OPEN ACCESS

Edited by:

Marian Elizabeth Ludgate,

Cardiff University, United Kingdom

Reviewed by:

Hector Manuel Targovnik, University of Buenos Aires, Argentina

Ulrich E. M. Schweizer,

University of Bonn, Germany

*Correspondence:

Yinlin Ge

geyinlin@yeah.net

†These authors have contributed equally to this work as co-first authors

Specialty section: This article was submitted to

Thyroid Endocrinology,

a section of the journal

Frontiers in Endocrinology

Received: 22 February 2019

Accepted: 16 July 2019

Published: 02 August 2019

Citation:

Liu S, Han W, Zang Y, Zang H Wang $F$, Jiang $P$, Wei $H$, Liu $X$,

Wang Y, Ma X and Ge Y (2019)

Identification of Two Missense Mutations in DUOX1 (p.R1307Q) and DUOXA1 (p.R56W) That Can Cause Congenital Hypothyroidism Through Impairing $\mathrm{H}_{2} \mathrm{O}_{2}$ Generation.

Front. Endocrinol. 10:526. doi: 10.3389/fendo.2019.00526

\section{Identification of Two Missense Mutations in DUOX1 (p.R1307Q) and DUOXA1 (p.R56W) That Can Cause Congenital Hypothyroidism Through Impairing $\mathrm{H}_{2} \mathrm{O}_{2}$ Generation}

\author{
Shiguo Liu ${ }^{1,2 t}$, Wenxiu Han ${ }^{3,4 t}$, Yucui Zang ${ }^{1,2}$, Hongwei Zang ${ }^{3}$, Fang Wang ${ }^{5}$, Pei Jiang ${ }^{4}$, \\ Hongwei Wei ${ }^{6}$, Xiangju Liu ${ }^{7}$, Yangang Wang ${ }^{5}, \mathrm{Xu} \mathrm{Ma}{ }^{8,9,10}$ and Yinlin $\mathrm{Ge}^{3 *}$ \\ ${ }^{1}$ Medical Genetic Department, The Affiliated Hospital of Qingdao University, Qingdao, China, ${ }^{2}$ Prenatal Diagnosis Center, The \\ Affiliated Hospital of Qingdao University, Qingdao, China, ${ }^{3}$ Department of Biochemistry and Molecular Biology, Medical \\ School of Qingdao University, Qingdao, China, ${ }^{4}$ Institute of Clinical Pharmacy, Jining First People's Hospital, Jining Medical \\ University, Jining, China, ${ }^{5}$ Department of Endocrinology and Metabolism, The Affiliated Hospital of Qingdao University, \\ Qingdao, China, ${ }^{6}$ Center of Newborn Screening, Linyi Women and Children Hospital, Linyi, China, ${ }^{7}$ Prenatal Diagnosis \\ Center, Taian Maternal and Child Health Hospital, Taian, China, ${ }^{8}$ Graduate School, Peking Union Medical College, Beijing, \\ China, ${ }^{9}$ Center for Genetic Eugenics, National Research Institute for Family Planning, Beijing, China, ${ }^{10}$ World Health \\ Organization Collaborating Center for Research in Human Reproduction, Beijing, China
}

Context: The DUOX/DUOXA systems play a key role in $\mathrm{H}_{2} \mathrm{O}_{2}$ generation in thyroid cells, which is required for iodine organification and thyroid hormone synthesis. DUOX2/DUOXA2 defects can cause congenital hypothyroidism $(\mathrm{CH})$, but it is unknown whether DUOX1/DUOXA1 mutations can also cause $\mathrm{CH}$.

Objective: We aimed to identify DUOX1/DUOXA1 mutations and explore their role in the development of $\mathrm{CH}$ by investigating their functional impacts on $\mathrm{H}_{2} \mathrm{O}_{2}$ generation.

Patients and Methods: Forty-three children with $\mathrm{CH}$ with goiter were enrolled, in whom all exons and flanking intronic regions of DUOX1/DUOXA1 were directly sequenced. We characterized the functional effects of identified mutations on the expression of DUOX1 and DUOXA1 and $\mathrm{H}_{2} \mathrm{O}_{2}$ generation.

Results: We identified a heterozygous DUOX1 missense mutation ( $G>A$ base substitution at nucleotide 3920 in exon 31) that changed a highly conserved arginine to glutamine at residual 1307 (p.R1307Q) in patient 1. A heterozygous-missense mutation (c.166 C>T; p.R56W) was identified in DUOXA1 in patient 2. Functional studies demonstrated that both p.R1307Q mutant or p.R56W mutant decreased the DUOX1 expression at mRNA and protein levels, with a corresponding impairment in $\mathrm{H}_{2} \mathrm{O}_{2}$ generation $(P<0.01)$. The results also showed that intact DUOXA1 was required for full activity of DUOX1 and $\mathrm{H}_{2} \mathrm{O}_{2}$ generation.

Conclusions: We have identified two heterozygous missense mutations in DUOX1 and DUOXA1 in two patients that can cause $\mathrm{CH}$ through disrupting the coordination of DUOX1 and DUOXA1 in the generation of $\mathrm{H}_{2} \mathrm{O}_{2}$. This study for the first time demonstrates that the DUOX1/DUOXA1 system, if genetically defective, can cause $\mathrm{CH}$.

Keywords: congenital hypothyroidism, DUOX1, DUOXA1, mutation, $\mathrm{H}_{2} \mathrm{O}_{2}$ generation 


\section{INTRODUCTION}

Congenital hypothyroidism $(\mathrm{CH})$ is the most common neonatal metabolic disorder which can result in abnormal growth and permanent mental retardation if treatment is delayed. Worldwide, $\mathrm{CH}$ affects 1 in 3,000-4,000 newborns and girls are more frequently affected than boys (female to male ratio is 2:1) (1). Most cases of $\mathrm{CH}$ are sporadic, and the occurrence of dyshormonogenesis caused by main enzyme defects in the thyroid hormone synthesis is generally inherited in an autosomal recessive manner and manifested as goitrous $\mathrm{CH}$ (2). Emerging evidence suggests that genetic defects in iodotyrosine deiodinase (IYD) (3), sodium-iodide symporter (NIS) (4), pendrin (SLC26A4) (5), thyroperoxidase (TPO) (6), thyroglobulin $(T G)(7)$, dual oxidase 2 (DUOX2) (8), and dual oxidase maturation factor 2 (DUOXA2) (9) can cause goitrous $\mathrm{CH}$, including transient or permanent types.

Hydrogen peroxide $\left(\mathrm{H}_{2} \mathrm{O}_{2}\right)$ plays a key role not only in iodine organification but also in tyrosine iodination and is thus critical to the synthesis of thyroid hormone (10). As members of the NADPH oxidase family, dual oxidases (DUOX1 and DUOX2) are supposed to be the sources of reactive oxygen species which can transfer one electron to iodine in iodine organification (11). Both DUOX1 and DUOX2 play a critical role in the production of $\mathrm{H}_{2} \mathrm{O}_{2}$ in the thyroid gland, which is the limiting factor in thyroglobulin iodination and thyroxine synthesis $(12,13)$. Interestingly, initial molecular studies demonstrated that no peroxide production could be detected even though DUOX family contains all characteristics consistent with NADPH oxidases (14). Subsequent studies demonstrated that DUOX enzymes were inactive due to its immature form inside the endoplasmic reticulum (ER) and only if the formation of heterodimeric complex consisting of DUOX and an additional partner, dual oxidase maturation factor (DUOXA), was accomplished, could the DUOX enzymatic activity be expressed (14). Thus, DUOXA is required for DUOX to form functional complexes, which is essential for ER-toGolgi transition, maturation and translocation to the plasma membrane. Only DUOX1/DUOXA1 and DUOX2/DUOXA2 complexes appear to be fully functional in vitro, whereas the DUOX2/DUOXA1 and DUOX1/DUOXA2 complexes appear to be unstable and tend to dissociate at the cell surface $(9,15)$.

Although increasing studies have demonstrated that DUOX2/DUOXA2 mutations are associated with $\mathrm{CH}$, it is unclear whether DUOX1/DUOXA1 defects can similarly cause $\mathrm{CH}$. Hoste et al. (16) reported some patients with complete inactivation of DUOX2 who manifested with transient $\mathrm{CH}$ early in life, suggesting that DUOX2 defects can be compensated fully by DUOX1 after the neonatal period is completed. However, no DUOX1 mutations and related genotypephenotype manifestations have been identified in goitrous $\mathrm{CH}$ patients. Moreover, the identification of novel DUOXA2 missense mutation in a transient $\mathrm{CH}$ patient with a large deletion comprising DUOX2, DUOXA2, and DUOXA1 (17) suggests that DUOXA1 mutation may lead to $\mathrm{CH}$ as DUOXA2 mutation does. Therefore, it is necessary to investigate the relationship between DUOX1/DUOXA1 defects and the development of
$\mathrm{CH}$. In the present study, we screened goitrous $\mathrm{CH}$ patients for DUOX1 and DUOXA1 mutations in a Chinese population, investigated the corresponding genotype-phenotype correlation, and characterized the functional effects of the identified mutations on DUOX1 and DUOXA1 at the molecular level.

\section{MATERIALS AND METHODS}

\section{Patients}

Forty-three (28 boys and 15 girls, male-female ratio 1.87:1.0, mean age $0.06 \pm 0.03$ years) cases of $\mathrm{CH}$ with goiter were identified in the neonatal screening at neonatal screening centers in Shandong province, China. The neonati were given full breast feeding $72 \mathrm{~h}$ after birth. Their heel blood was dropped in a dry blood spot on a filter paper to examine thyroid stimulating hormone (TSH) concentration for screening. Thyroxine (T4), triiodothyronine (T3), TSH, free thyroxine (FT4), and free triiodothyronine (FT3) in the serum were formally measured when the subjects had elevated TSH $(\geq 20 \mu \mathrm{IU} / \mathrm{ml})$ levels on the screening test. The subjects were diagnosed with $\mathrm{CH}$ if they had abnormally elevated TSH concentration (normal range $0.27-$ $4.2 \mu \mathrm{IU} / \mathrm{ml}$ ) and low FT4 concentration (normal range 12-22 $\mathrm{pmol} / \mathrm{L}$ ). This biochemical diagnosis of $\mathrm{CH}$ was followed by a $99 \mathrm{mTc}$ thyroid scan or thyroid ultrasound examination in the patients with eutopic thyroid gland. All the subjects from 43 unrelated families were free from other congenital diseases. Our study was approved by the medical ethics committee of the Affiliated Hospital of Qingdao University with informed consent of patient families.

\section{DNA Analysis of DUOX1 and DUOXA1}

Genomic DNA was isolated from peripheral blood leucocytes of patients and stored at $-20^{\circ} \mathrm{C}$ up to the time of analysis. The complete sequences of DUOX1 (35 exons) and DUOXA1 (11 exons), including splice and flanking intronic regions, were amplified by polymerase chain reaction (PCR). The primer sequences of all exons in DUOX1 and DUOXA1 are listed in Tables 1, 2, respectively. Mutations in DUOX2 (10), DUOXA2 (9), TPO, TG, and NIS (18) for all subjects had been excluded in our previous study in these patients. The PCR reaction system $(25 \mu \mathrm{L})$ consisted of $100 \mathrm{ng}$ of genomic DNA, $1 \mathrm{U}$ of Taq DNA polymerase, $500 \mu \mathrm{M}$ dNTPs, $0.5 \mu \mathrm{M}$ each primer and $1 \times$ reaction buffer ( $20 \mathrm{mM}$ Tris- $\mathrm{HCl}, \mathrm{pH} 8.0,3 \mathrm{mM} \mathrm{MgCl}_{2}$ ). Samples were pre-denatured at $95^{\circ} \mathrm{C}$ for $5 \mathrm{~min}$, followed by PCR reaction for 35 cycles of $30 \mathrm{~s}$ at $95^{\circ} \mathrm{C}, 30 \mathrm{~s}$ at $55-61^{\circ} \mathrm{C}$, and $30 \mathrm{~s}$ at $72^{\circ} \mathrm{C}$, with a final extension at $72^{\circ} \mathrm{C}$ for $7 \mathrm{~min}$. Purified PCR products were analyzed on $2 \%$ agarose gel electrophoresis and mutations were identified by analyzing the nucleotide sequence (NC_000015.10) after ABI 3730XL (Applied Biosystems) sequencing.

\section{cDNA and Construction of DUOX1/DUOXA1 Expression Vector}

After extraction of RNA from human thyroid tissue, cDNA was synthesized by the primer oligo (dT) through the reverse transcription reaction. The consensus coding sequence of DUOX1 (4,656 bp) was amplified from cDNA (forward primer: 5'-ATGGGCTTCTGCCTGGCTCTA-3', reverse primer: 5' -CTA 
TABLE 1 | The primer sequences of all exons of DUOX1 gene.

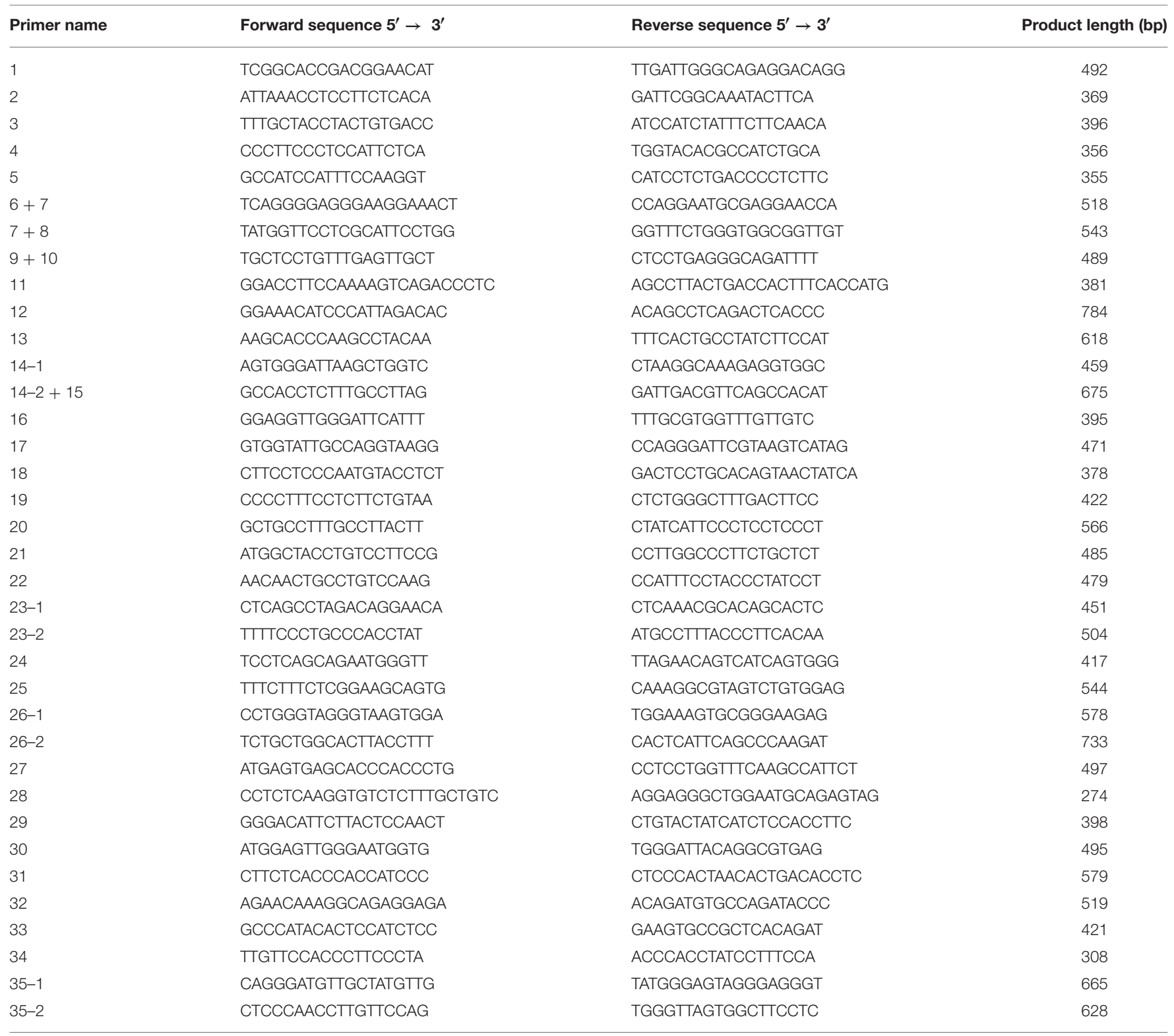

DUOX1, dual oxidase 1.

GAAGTTCTCATAATGGTG-3') and was cloned into pEASYBlunt M2 expression vector. Similarly, the consensus coding sequence of DUOXA1 (1,452 bp) was amplified from cDNA (forward primer: 5'-ATGGCTACTTTGGGACACACA-3', reverse primer: $5^{\prime}$-TCAGATTAGAGGTGTGTGGCG-3') and was cloned into pEASY-Blunt M2 expression vector. DUOX1 p.R1307Q mutant was introduced by site-directed mutagenesis (Fast Mutagenesis System) into the expression vector using the mutagenic forward primer 5'-GAGCGGCCAGTGGGTCC AAATCGCTTGTC- $3^{\prime}$ and the mutagenic reverse primer $5^{\prime}$-TGG ACCCACTGGCCGCTCTTGTACTCGAA-3'. The expression vector of mutant DUOXA1 p.R56W was constructed on the basis of wild type expression vector using the mutagenic forward primer $\quad 5^{\prime}$-GGCTGTTCTGGCTGCTTTGGGTGGTGACC-3' and the mutagenic reverse primer 5'-AAAGCAGCCAGAAC AGCCTCGTCTTTCCC- $3^{\prime}$ in the same way. All the constructed expression vectors were confirmed by DNA sequencing.

\section{Cell Culture and Transient Cell Transfection}

HeLa cells were grown in six-well plates in 1640 culture medium supplemented with $10 \%$ fetal calf serum in a humidified condition with $5 \% \mathrm{CO}_{2}$ at $37^{\circ} \mathrm{C}$. HeLa cells were divided into eight groups according to the transfected expression vector type before transfection, including DUOX1 (containing DUOX1 wild type [WT] expression vector only), DUOXA1 (containing DUOXA1 WT expression vector only), DUOX1 and DUOXA1 (containing both DUOX1 WT and DUOXA1 WT expression vector), p.R1307Q and DUOXA1 (containing 
TABLE 2 | The primer sequences of all exons of DUOXA1 gene.

\begin{tabular}{|c|c|c|c|}
\hline Primer name & Forward sequence $\left(5^{\prime} \rightarrow 3^{\prime}\right)$ & Reverse sequence $\left(5^{\prime} \rightarrow 3^{\prime}\right)$ & Product length (bp) \\
\hline 1 & AGCCCTCCСAAATCTGACCT & GGCACCGACGGAACATCTC & 316 \\
\hline 2 & TCCGCCTTCACAAGTCCC & AGCTCCAGCGCAAACCTAG & 312 \\
\hline 3 & TCTGAGAAGTTGGGAGTGAC & TCTGGATGAAAGCAGGAAGT & 405 \\
\hline 4 & GCAGTGGAACGGTGGTAA & СTCCTGGGCTCAAGCAAT & 590 \\
\hline 5 & TAGCAGAGTCTGATGATGCACAAA & CCACCAGCGCTCAATAGTGA & 500 \\
\hline 8 & GGGTAGAAACCCTGTTCCTGAA & GGGAGAGAAAATCAGGAGATAAGAGA & 501 \\
\hline $9-1$ & TAGGCTTCCCTTAGAGTTGTTCTGA & TTGGCCTTATCATGGCAACAG & 600 \\
\hline $9-2$ & CCTCAGGGTGGCTGTTACCA & TTCCCACCTGGCTTCTTGTG & 600 \\
\hline $9-3$ & TTCCCACCTGGCTTCTTGTG & CCAGACTTAAAATGTATCACCACTAACC & 677 \\
\hline $9-4$ & CCAGACTTAAAATGTATCACCACTAACC & TGCACTTCCAGTTACAGAATGAA & 642 \\
\hline
\end{tabular}

DUOXA1, dual oxidase maturation factor 1.

DUOX1 p.R1307Q mutant expression vector and DUOXA1 WT expression vector), DUOX1 and p.R56W (containing DUOX1 WT expression vector and DUOXA1 p.R56W mutant expression vector), p.R1307Q and p.R56W (containing both DUOX1 p.R1307Q and DUOXA1 p.R56W mutant expression vectors), pEASY-Blunt M2 (containing an empty vector), and parental non-transfected cells (containing no expression vector). When HeLa cells reached $70-80 \%$ confluence, they were transfected with $4 \mu \mathrm{g}$ of plasmid DNA (WT, mutant, and control) in sixwell plates using TransIn EL Transfection Reagent (TransGen, Beijing, China). To ensure quantitative consistency of plasmids, empty plasmid was used as a reference in individual transfected groups and the DUOX1/DUOXA1 ratio was 7:1. The culture medium was replaced at $6 \mathrm{~h}$ after transfection and cells were harvested after $48 \mathrm{~h}$.

\section{mRNA Expression of DUOX1 and DUOXA1}

HeLa cells cultured on six-well plates were suspended in Trizol reagent (Invitrogen, Carlsbad, USA) and total RNA was purified $48 \mathrm{~h}$ after transfection. Reverse transcription was performed using a Prime Script $\left({ }^{\mathrm{TM}}\right)$ RT Enzyme Mix I (TaKaRa, Shiga, Japan) on $1 \mu \mathrm{g}$ of total RNA in a reaction volume of $20 \mu \mathrm{L}$. One-fifth of the cDNA product was used for real time PCR amplification using TIANGEN Taq DNA polymerase (TIANGEN, Beijing, China) and the amplified PCR products were detected according to the fluorescence intensity of SYBR Green I. For specific amplification of human DUOX1 cDNA, forward primer was 5'-TCTGGGACTGCTCTGGTTC$3^{\prime}$ and reverse primer $5^{\prime}$-CGATGTTCTGGTAGGTGGC-3'; for human DUOXA1, forward primer was $5^{\prime}$-AAAGGCTCTGGA GAAGGG-3' and reverse primer 5'-GCATGGCTGAGGTGT AGTG-3' ; for GAPDH (loading control), forward primer was $5^{\prime}$ AGAAGGCTGGGGCTCATTTG-3' and reverse primer $5^{\prime}$-AGG GGCCATCCACAGTCTTC-3'. Samples were heated to $95^{\circ} \mathrm{C}$ for $15 \mathrm{~min}$, followed by 40 cycles of $10 \mathrm{~s}$ at $95^{\circ} \mathrm{C}, 32 \mathrm{~s}$ with annealing/elongation at $63^{\circ} \mathrm{C}$. The amplified level of the target gene was normalized against that of GAPDH.

\section{Western Blot Analysis of DUOX1 Expression}

After transfection for $48 \mathrm{~h}$, monolayer cells were suspended in cell lysis solution containing protease inhibitors (Phenyl methane sulfonyl fluoride, PMSF) on ice for $30 \mathrm{~min}$ after washing with physiological saline. Total protein was collected from the supernatant of cell lysates after centrifugation for $20 \mathrm{~min}$ at $4^{\circ} \mathrm{C}$. After measurement of total protein concentration using a BCA Protein Quantitative Kit (TransGen, Beijing, China), protein samples $(30 \mu \mathrm{g})$ were denatured by heating water at $95^{\circ} \mathrm{C}$ for $10 \mathrm{~min}$ in the loading buffer (TransGen, Beijing, China), subjected to SDS-PAGE using a $8 \%$ acrylamide mini-gel, and transferred to a PVDF membrane. The PVDF membrane was blocked for $2 \mathrm{~h}$ at room temperature with 5\% nonfat dry milk in PBS (pH7.4) and 0.1\% Tween 20 and was then incubated overnight at $4{ }^{\circ} \mathrm{C}$ with primary antibodies against DUOX1 (Bioss, Beijing, China) at a 1:250 dilution and against beta-Actin (Loading Control) at a 1:2,000 dilution in PBSTween 20 containing 5\% nonfat dry milk. Membranes were washed, incubated for $1 \mathrm{~h}$ with peroxidase-labeled secondary antibody anti-rabbit, washed again, and visualized with enhanced chemiluminescence on CL-XPosure films.

\section{Measurement of $\mathrm{H}_{\mathbf{2}} \mathrm{O}_{2}$ Generation}

Extracellular $\mathrm{H}_{2} \mathrm{O}_{2}$ generation was quantified by the Amplex red/horseradish peroxidase assay (Molecular Probes, Invitrogen), which detected the accumulation of a fluorescent oxidized product. Amplex Red reagent (10-acetyl3,7-dihydroxyphenoxazine, $50 \mu \mathrm{M})$ and horseradish $(0.1 \mathrm{U} / \mathrm{ml})$ peroxidase (HRP) was added into each well after transfection for $48 \mathrm{~h}$ and the six-well plate was placed in an incubator with dark environment at $37^{\circ} \mathrm{C}$ for $1 \mathrm{~h}$. The fluorescence intensity was measured by Synergy $\left({ }^{\mathrm{TM}}\right)$ multi-functional enzyme marking 
instrument using excitation at $535 \mathrm{~nm}$ and emission at $595 \mathrm{~nm}$. $\mathrm{H}_{2} \mathrm{O}_{2}$ release of experiment groups and control groups were quantified using standard calibration curves (as shown in Supplementary Figure 1) which could change the fluorescence intensity into absolute nanomoles of $\mathrm{H}_{2} \mathrm{O}_{2}$.

\section{Protein Synthesis Suppression Assay}

Cycloheximide $(\mathrm{CHX})$ at $100 \mu \mathrm{g} / \mathrm{ml}$ was added to block the synthesis of intracellular protein and total protein was extracted after cells were disassociated in four different time periods within $24 \mathrm{~h}$. The DUOX1 protein expression was detected by Western blot.

\section{Statistical Analysis}

Statistics analysis of variance (ANOVA) was used for data presented as mean \pm standard deviation (SD) using the software of SPSS 17.0, followed by LSD (least significant difference) multiple comparison test. A $P<0.05$ was considered to be statistically significant.

\section{RESULTS}

\section{Mutation Screening and Genotype-Phenotype Analysis}

A heterozygous missense mutation (c.3920G $>$ A, present in Genome Aggregation Database, Figure 1A) was identified in the DUOX1 gene in patient 1 (P1), causing an arginine-toglutamine substitution at amino acid 1307 of DUOX1, which was located in a conserved sequence of the protein according to the multiple sequence alignment analysis (Figure 2A). This mutation was not found in 100 healthy control Chinese subjects. The relationship was not clear between DUOX1 mutation segregation

\section{CAG G G CAG TG G G TG CG G A T CG C T TG C C TG G} 50 60 70

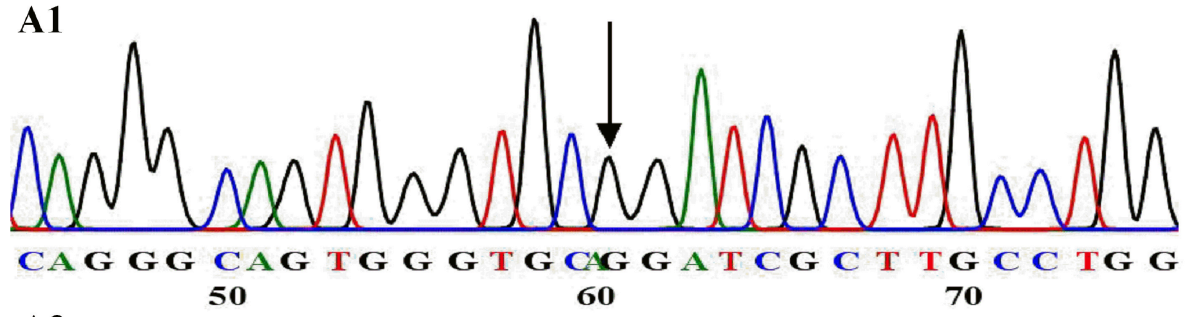
A2

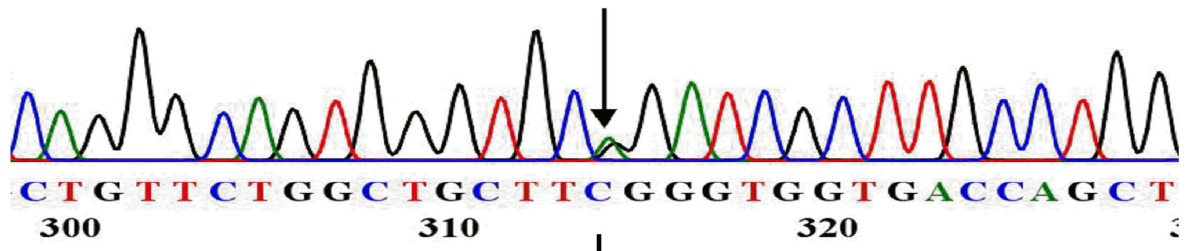

B1

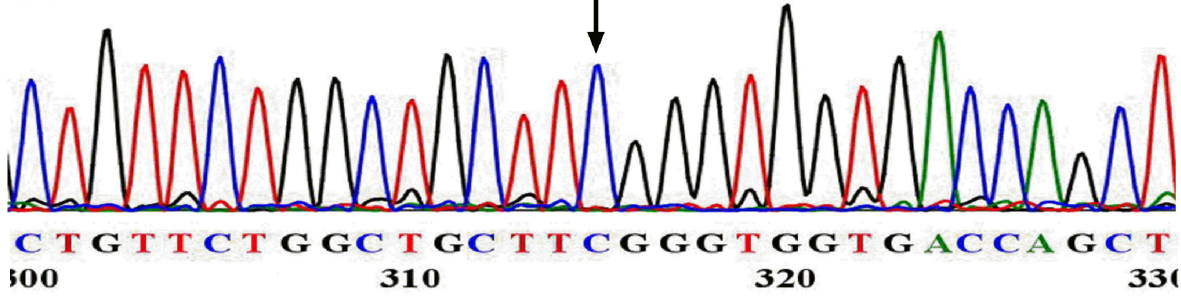

B2

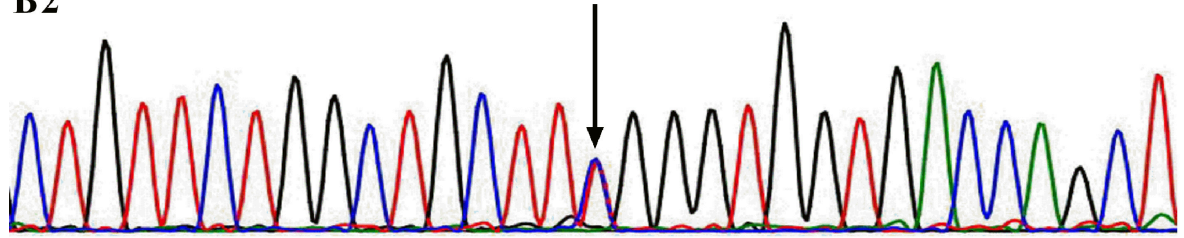

FIGURE 1 | WT and mutational sequences of exon 31 in DUOX1 (A) and exon 6 in DUOXA1 (B). (A1) Arrowhead indicates homozygous G at nucleotide 3920 in normal individuals; (A2) Arrowhead indicates the heterozygous A and $\mathrm{G}$ at nucleotide 3920 in patient 1. (B1) Arrowhead indicates homozygous $\mathrm{C}$ at nucleotide 166 in normal individuals; (B2) Arrowhead indicates the heterozygous C and T at nucleotide 166 in patient 2. 
and phenotype within the family because of the lack of relevant data. P1 was a female infant with birth weight of $2,500 \mathrm{~g}$. She was born at full-term gestation by cesarean delivery and had no family history of thyroid disease. She was called back to hospital at 36 days (body weight 3,800 g, height $47 \mathrm{~cm}$ ) after birth for further evaluation because of her high TSH concentration ( $>100 \mu \mathrm{IU} / \mathrm{ml}$ ) on the filter paper neonatal screening test. Her thyroid gland was enlarged on the $99 \mathrm{mTc}$ thyroid scan and the perchlorate discharge test was positive. Levothyroxine (L-T4) replacement therapy was started immediately with the initial dose of $7.7 \mu \mathrm{g} / \mathrm{kg}$. To maintain normal serum TSH and FT4 levels, replacement therapy dose was adjusted based on her clinical and hormonal evaluations during the subsequent follow-up. TSH, FT3, and FT4 levels remained normal following a temporary withdrawal of L-T4 therapy for 4 weeks when she was 2 years old. Her final diagnosis was therefore transient $\mathrm{CH}$. The patient is currently 10.5 years old with normal physique and intelligence.

Patient 2 (P2) carried a DUOXA1 heterozygous missense mutation p.R56W (a C>T transversion at nucleotide 166 in exon 6, present in Genome Aggregation Database, Figure 1B) that changes the highly conserved arginine at amino acid 56 of DUOXA1 protein (Figure 2B) to tryptophan and resulted in severe congenital hypothyroidism, which was not identified in 100 healthy control Chinese subjects. P2 was a male subject (birth weight $2,750 \mathrm{~g}$ ) with a p.R56W mutation, born by normal delivery at full-term gestation. He was called back to hospital after neonatal screening at 35 days from birth for a high level of TSH $(>100 \mu \mathrm{IU} / \mathrm{ml})$ on the filter paper screening test; he was subsequently diagnosed with $\mathrm{CH}$ with a low serum FT4 level (4.7 pmol/L). A 99mTc thyroid scan indicated that P2 had eutopic thyroid gland with mild diffuse goiter. L-T4 replacement therapy was started immediately at an initial dose of $8.4 \mu \mathrm{g} / \mathrm{kg}$ per day. To maintain normal serum TSH, FT3, and FT4 levels, the dose was adjusted according to clinical and hormonal evaluations. The patient continued to have hypothyroidism requiring thyroid hormone therapy at 2.5 years; this patient was therefore diagnosed with permanent $\mathrm{CH}$. The patient is currently 9.5 years old and his physical and mental developments are normal. She currently receives L-T4 with a daily dose of $5.9 \mu \mathrm{g} / \mathrm{kg}$.

\section{Effects of Mutations on the Expression of DUOX1/DUOXA1}

The relative expression of DUOX1 mRNA decreased with mutant constructs in comparison with both wild type constructs $(P<$ 0.01), and the group with p.R1307Q and p.R56W combination (p.R1307Q + p.R56W) had the least expression of DUOX1 mRNA compared with other groups $(P<0.01)$. With respect to the relative expression of DUOXA1 mRNA, groups with p.R56W mutant (DUOX1 + p.R56W or p.R1307Q + p.R56W) were far lower than other groups except for the group with DUOX1 only $(P<0.01)$ (Figure 3).

Consistent with the results of mRNA expression, both p.R1307Q and p.R56W were associated with decreased expression of DUOX1 protein compared with the wild type protein on the western blot analysis $(P<0.01)$. In particular, the expression of DUOX1 protein in HeLa cells transfected with both p.R1307Q and p.R56W in combination decreased most significantly $(P<0.01)$ (Figure 4$)$. The size of the bands of DUOX1 protein is presented in Supplementary Figure 2.

\section{$\mathbf{H}_{\mathbf{2}} \mathrm{O}_{\mathbf{2}}$ Production Assay}

As shown in Figure 5, transfection with wild-type DUOX1 or DUOXA1 each alone significantly increased $\mathrm{H}_{2} \mathrm{O}_{2}$ production compared with empty vector transfection. Transfection with mutant p.R1307Q or p.R56W alone resulted in reduced $\mathrm{H}_{2} \mathrm{O}_{2}$ production compared with their corresponding wild-type counterparts, respectively $(P<0.01)$. The group transfected

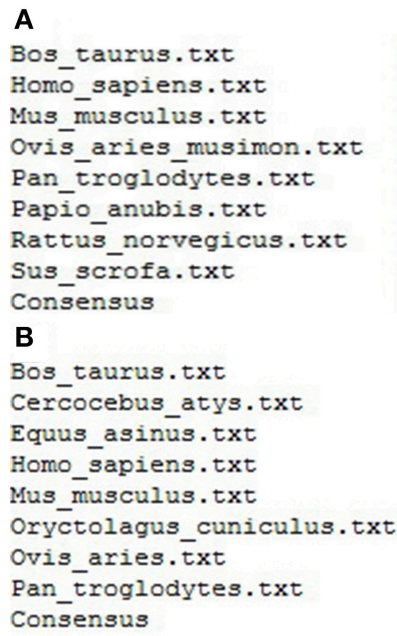

A

Bos taurus.txt

Homo_sapiens.txt

Mus musculus.txt

Ovis_aries_musimon.txt

Pan troglodytes.txt

Papio_anubis.txt

Rattus norvegicus.txt

Sus_scrofa.txt

Consensus

B

Bos taurus.txt

Cercocebus_atys.txt

Equus asinus.txt

Homo sapiens.txt

Mus musculus.txt

Oryctolagus_cuniculus.txt

Ovis aries.txt

Pan_troglodytes.txt

Consensus

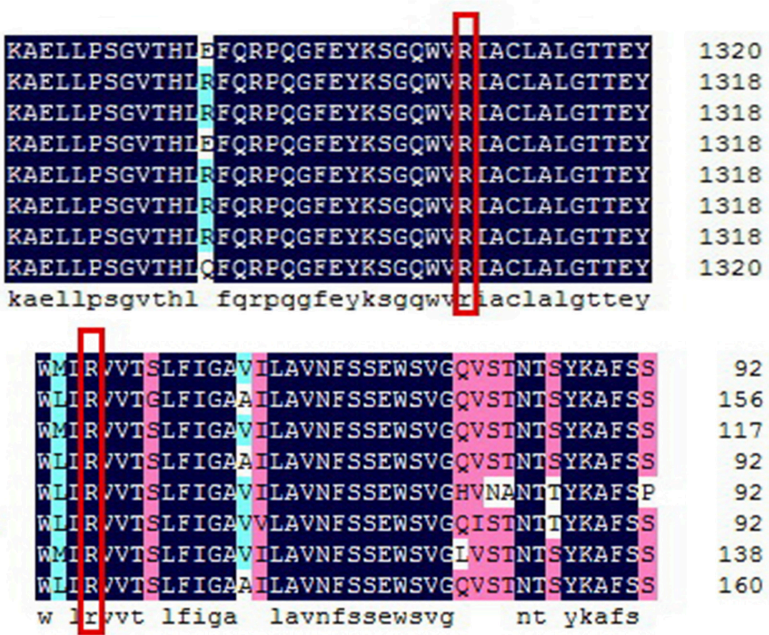

FIGURE 2 | Sequence alignment analysis of DUOX1 (A) and DUOXA1 (B) in different species. (A) Red rectangle indicates arginine at amino acid 1307of DUOX1 located in a conserved sequence of the DUOX1 protein. (B) Red rectangle indicates arginine at amino acid 56 of DUOXA1 located in a conserved sequence of the DUOXA1 protein. 


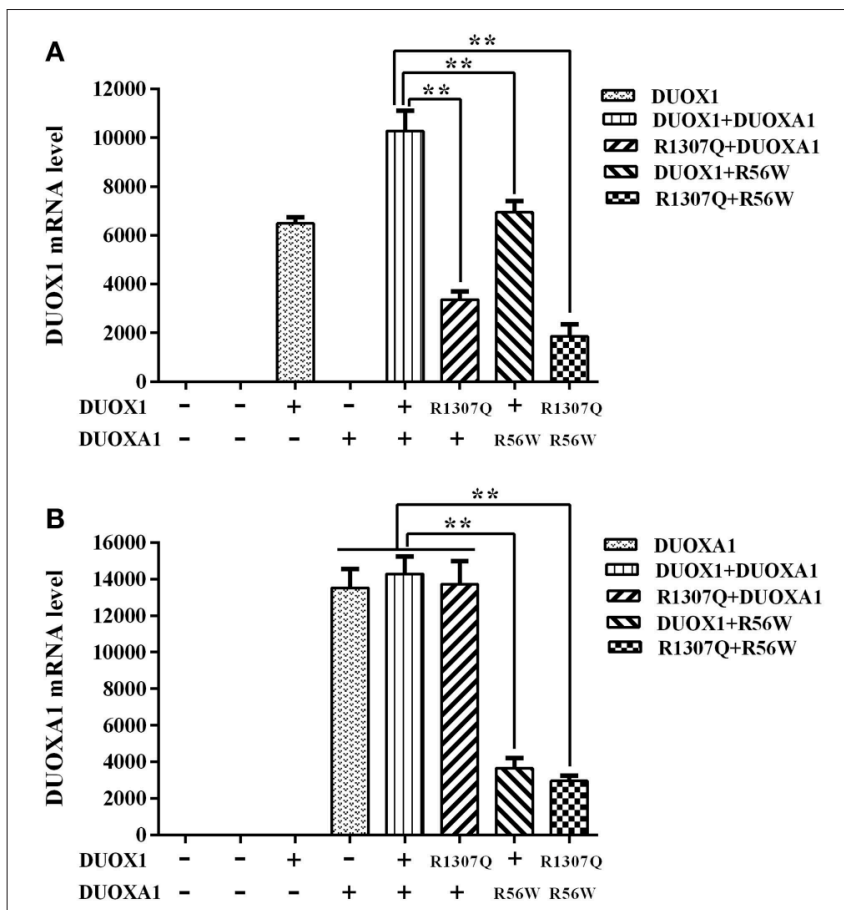

FIGURE 3 | Relative mRNA expression levels under the various indicated vector transfections. (A) DUOX1 mRNA expression. The DUOX1 mRNA level was highest in the group with both wild-type DUOX1 and DUOXA1 expression vectors, while the group with $\mathrm{p} . \mathrm{R} 1307 \mathrm{Q}$ and $\mathrm{p}$.R56W combination had the least mRNA expression of the DUOX1 gene compared with other experimental groups except for the group with DUOXA1 only. (B) DUOXA1 mRNA expression. Groups with p.R56W mutant were far lower than other experimental groups with DUOXA1. Each column represents the mean $\pm \mathrm{SD}$ of three independent experiments performed in triplicate. ${ }^{\star \star} P<0.01$.

with both wild-type DUOX1 and DUOXA1 in combination (DUOX1 or DUOXA1) produced the most amount of $\mathrm{H}_{2} \mathrm{O}_{2}$ $(P<0.01)$ while, in contrast, the group with transfection of both mutants p.R1307Q and p.R56W in combination (p.R1307Q + p.R56W) impaired the $\mathrm{H}_{2} \mathrm{O}_{2}$-generating system to the maximum extent $(\mathrm{P}<0.01)$. These patterns were consistent with the Western blot analysis results above (Figure 4). Thus, these DUOX1 and DUOXA1 mutants were able to substantially inhibit the functional activities of the $\mathrm{H}_{2} \mathrm{O}_{2}$-generating system, demonstrating the functional consequences of the two mutations.

\section{Effects of CHX on DUOX1 Expression}

DUOX1 protein synthesis with transfection of all expression vectors for $24 \mathrm{~h}$ was blocked with the addition of CHX. Compared with the quantity of DUOX1 protein at the beginning of adding CHX $(0 \mathrm{~h})$, the degradation rate of mutant DUOX1 protein had no significant difference compared with wild type DUOX1 protein at 6, 12, and $24 \mathrm{~h}$ after transfection $(P>0.05$; Figure 6).

\section{DISCUSSION/CONCLUSION}

A crucial step in thyroid hormone biosynthesis is iodine organification, which can only be successfully accomplished in the presence of $\mathrm{H}_{2} \mathrm{O}_{2}$. The NADPH oxidases DUOX1 and DUOX2, also known as thyroid oxidases ThOX1 and ThOX2, were originally identified in the thyroid tissue thanks to the cloning of the human cDNA encoding NADPH oxidase (12, 13). The two corresponding genes (DUOX1 and DUOX2) are located on chromosome 15q15.3 and encode 1,551 and 1,548 amino acids in length, respectively. As seventh-pass-membrane proteins, DUOX proteins are composed of the extracellular peroxidase-like domain, transmembrane domain, two EF-hand domains, one FAD-binding site, and four nicotinamide adenine dinucleotide phosphate (NADPH)-binding sites (13, 19-21). As a candidate thyroid $\mathrm{H}_{2} \mathrm{O}_{2}$ generator, the activity of DUOX is likely to adapt to the feedback of the local $\mathrm{H}_{2} \mathrm{O}_{2}$ concentrations (22, 23). Recent studies showed that the activation of DUOX-based $\mathrm{H}_{2} \mathrm{O}_{2}$ generator relies on the formation of a heterodimer complex consisting of DUOX and a specific maturation factor (DUOXA1 and DUOXA2) (24). DUOXA1 and DUOXA2 were first cloned and named in Chicago in 2006 (25). They are located on chromosome 15 and consist of 6 (DUOXA2) and 11 (DUOXA1) exons, encoding transmembrane proteins of 320 (DUOXA2) and 483(DUOXA1) amino acids, respectively. Other than the thyroid tissue, DUOXA2 can be detected in the epithelium of the digestive tract at low levels (26).

The finding of the association of DUOX2/DUOXA2 system with $\mathrm{CH}$ led to the identification of their predominant role in thyroid hormone synthesis. It is well-known that DUOX2/DUOXA2 mutations can cause $\mathrm{CH}$ directly, while it is controversial whether the DUOX1/DUOXA1 system can also play a role in $\mathrm{CH}$. It was previously believed that DUOX1 had no relationship with $\mathrm{CH}$ due to the fact that DUOX2 deficiency could result in severe hypothyroidism, indicating that DUOX1 could not compensate the deficiency and was therefore not important in thyroid hormone synthesis (27). In addition, DUOX1 knockout mice had normal growth and serum thyroxin levels, supporting this belief. Nevertheless, the above hypothesis was questioned by later studies which demonstrated that DUOX1 participated partially in thyroid hormone synthesis when complete loss of DUOX2 activity occurred (28). Moreover, the clinical identification of a patient with Y246X homozygous nonsense mutation in DUOXA2 also suggested such a function of DUOXA1 under the circumstances of DUOXA2 inactivation (9). Therefore, it is interesting and important to explore the role of the DUOX1/DUOXA1 system in $\mathrm{CH}$.

In the present study, we found that $\mathrm{P} 1$ with $\mathrm{CH}$ with goiter carried a heterozygous missense mutation (c.3920G $>$ A, p.R1307Q) in DUOX1. The highly conserved amino acid arginine coded by the wild type codon where the mutation occurred is localized in the intracellular domain of $\mathrm{C}$ terminal near the FAD binding domain. Clinical data indicated that this patient indeed had a partial defect of $\mathrm{H}_{2} \mathrm{O}_{2}$ generation in the DUOXA1 reconstitution assay and manifested biochemically as a transient form of $\mathrm{CH}$ in the neonatal and infantile period. Subsequent studies showed that the requirements of thyroid hormone synthesis reached highest at the neonatal period (10$15 \mathrm{~g} / \mathrm{kg})$, which is five to seven times that of an adult $(2 \mathrm{~g} / \mathrm{kg})$ (29). In contrast, P2 with DUOXA1 p.R56W mutation, occurring at a highly conserved position among different species and 
A
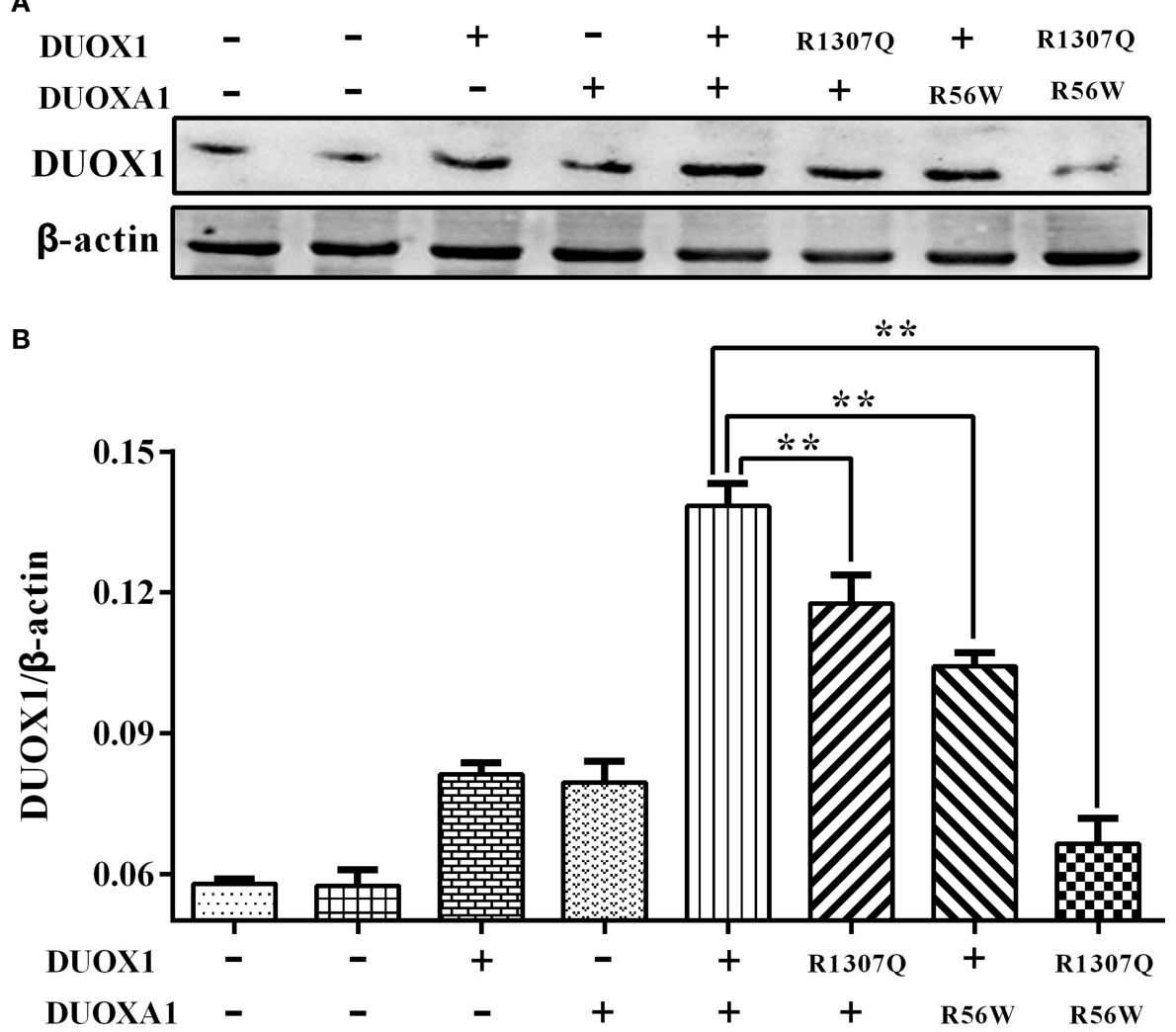

FIGURE 4 | Western blot analysis of the DUOX1 protein expression under various indicated vector transfections. (A) Representative presentation of the gel results. The DUOX1 protein level was highest in the group with both wild-type DUOX1 and DUOXA1 expression vectors, while the group transfected with both p.R1307Q mutant and p.R56W mutant had the least expression level. (B) Densitometric measurement of DUOX1 protein expression. The results are presented as the ratio of DUOX1/B-actin, corresponding to (A). Primary antibodies against DUOX1 was used at a 1:250 dilution and Anti-beta-actin, as loading control, was used at a 1:2,000 dilution. Each column represents the mean $\pm \mathrm{SD}$ of three independent experiments performed in triplicate. ${ }^{\star \star} P<0.01$.

resulting in DUOX1 protein being hampered by the failure to reconstitute active DUOX enzymes, manifested with permanent $\mathrm{CH}$. Although the amount of DUOX2 expression was about five times more than DUOX1 and the DUOX2/DUOXA2 system was the primary source of $\mathrm{H}_{2} \mathrm{O}_{2}$ generation, the p.R56W mutant could cause a serious deficiency of thyroid hormone synthesis and eventually permanent $\mathrm{CH}$. Our study indicated that P2 with permanent $\mathrm{CH}$ can be caused by monoallelic heterozygous missense DUOXA1 mutation, which was consistent with previous results showing that biallelic (9), and possibly also monoallelic (30), DUOXA2 mutations could cause permanent $\mathrm{CH}$ with partial iodide organification defect.

$\mathrm{H}_{2} \mathrm{O}_{2}$ generation experiment showed that p.R1307Q near the FAD binding domain impaired DUOX1 enzyme activity, while cycloheximide ( $\mathrm{CHX}$ ) chase experiment showed that the stability of DUOX1 protein was not changed based on the DUOX1 protein level at half-time (the half-life is $6 \mathrm{~h}$ predicted by ProtScale), which was consistent with the stability prediction by ProtScale, a bio-software which predicted that p.R1307Q mutant could not change the DUOX1 protein stability. After transfection with the DUOXA1 expression vector, the group transfected with both p.R1307Q and p.R56W expression vector had the least amount of $\mathrm{H}_{2} \mathrm{O}_{2}$ generation. In contrast, the group transfected with both wild type DUOX1 and wild type DUOXA1 expression vectors had the largest amount of $\mathrm{H}_{2} \mathrm{O}_{2}$ generation. Moreover, the group with both wild type constructs had the maximum DUOX1 mRNA level and DUOX1 mRNA level decreased significantly in the group with p.R56W mutant vectors. As mentioned above, DUOXA is necessary for DUOX to form functional complexes to exert its biological function, hypothesis on the decreased DUOX1 mRNA level caused by p.R56W DUOXA1 mutation are as follows: (1) p.R56W DUOXA1 mutation may participate in the transcriptional regulation, including transcriptional initiation and termination, and thus result in the decrease of DUOX1mRNA level. (2) p.R56W DUOXA1 mutation may affect the DUOX1 mRNA stability through destroy the structure of $3^{\prime}$ poly(A) tail and 3' UTR, and consequently cause the degradation of DUOX1 mRNA. Consistently, the group with both WT expression vectors had the maximum DUOX1 protein expression and the group with both mutant expression vectors had the minimum DUOX1 protein expression. Functional studies indicated that the p.R1307Q mutant caused both partial functional loss of the DUOX1 activity and the protein expression. Moreover, 


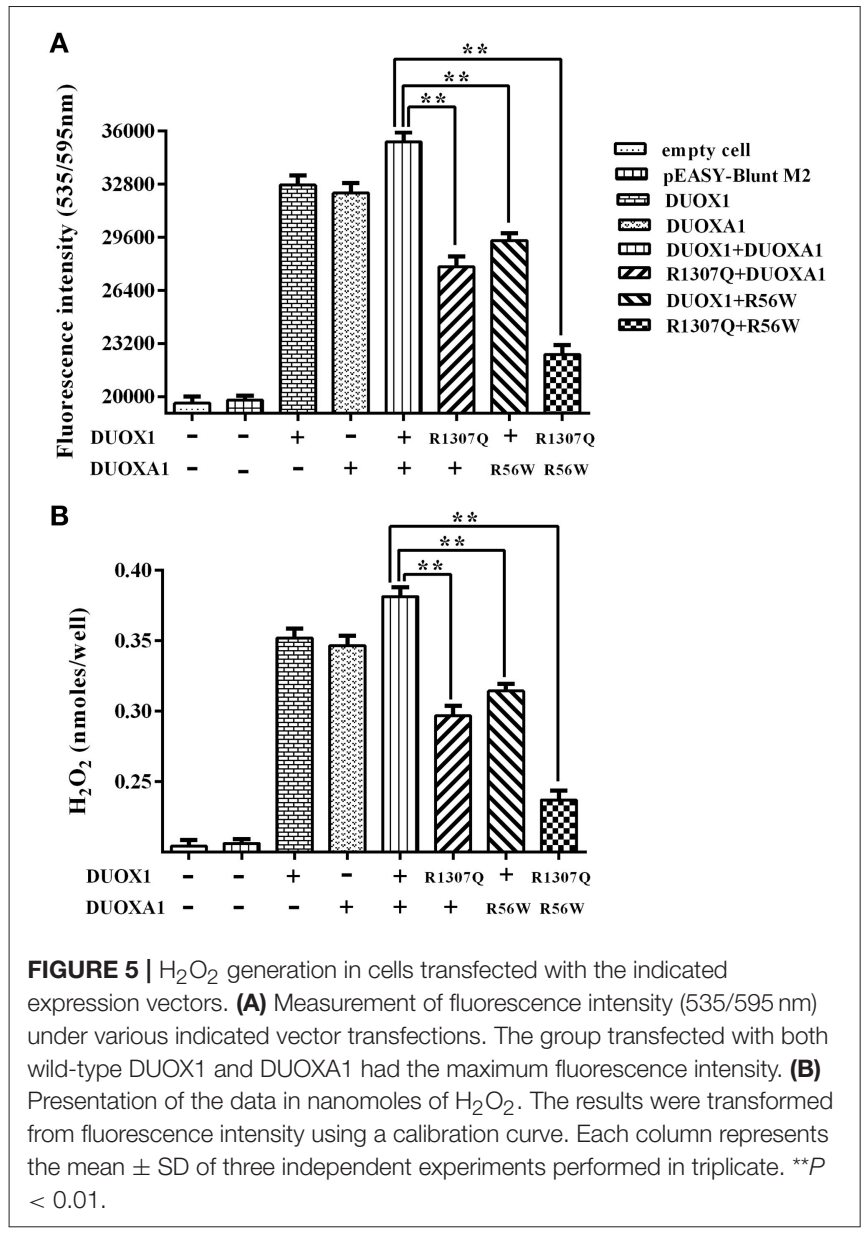

introduction of the p.R56W mutant could impair the DUOX1 activity and the protein expression more profoundly, suggesting that DUOXA1 was necessary for DUOX1 to perform normal function. Consequently, these data, taken together, make it plausible to propose that the DUOX1/DUOXA1 system, when mutated, can cause $\mathrm{CH}$.

In our study, patient 1 and patient 2 each only had one mutation even after screening on all exons of DUOX1 (P1) and DUOXA1 (P2), respectively. Based on the functional results in the present study and published data, the development of $\mathrm{CH}$ in the two patients could be mechanistically explained as follows: (1) Monoallelic DUOX1/DUOXA1 mutations may cause CH just like DUOX2 (31)/DUOXA2 (30); (2) Intact DUOX1 is needed for full function of the $\mathrm{H}_{2} \mathrm{O}_{2}$ generating system and intact DUOXA1 is needed for maximal activity of DUOX1; (3) There may be concurrent genetic alterations such as mutations of relevant genes haven't been examined in the two patients. Future studies are needed to elucidate the specific molecular mechanisms involved.

In conclusion, we have identified two heterozygous missense mutations in DUOX1 and DUOXA1 in two Chinese patients with $\mathrm{CH}$ with goiter, respectively. Functional studies demonstrate that the two mutations each can partially

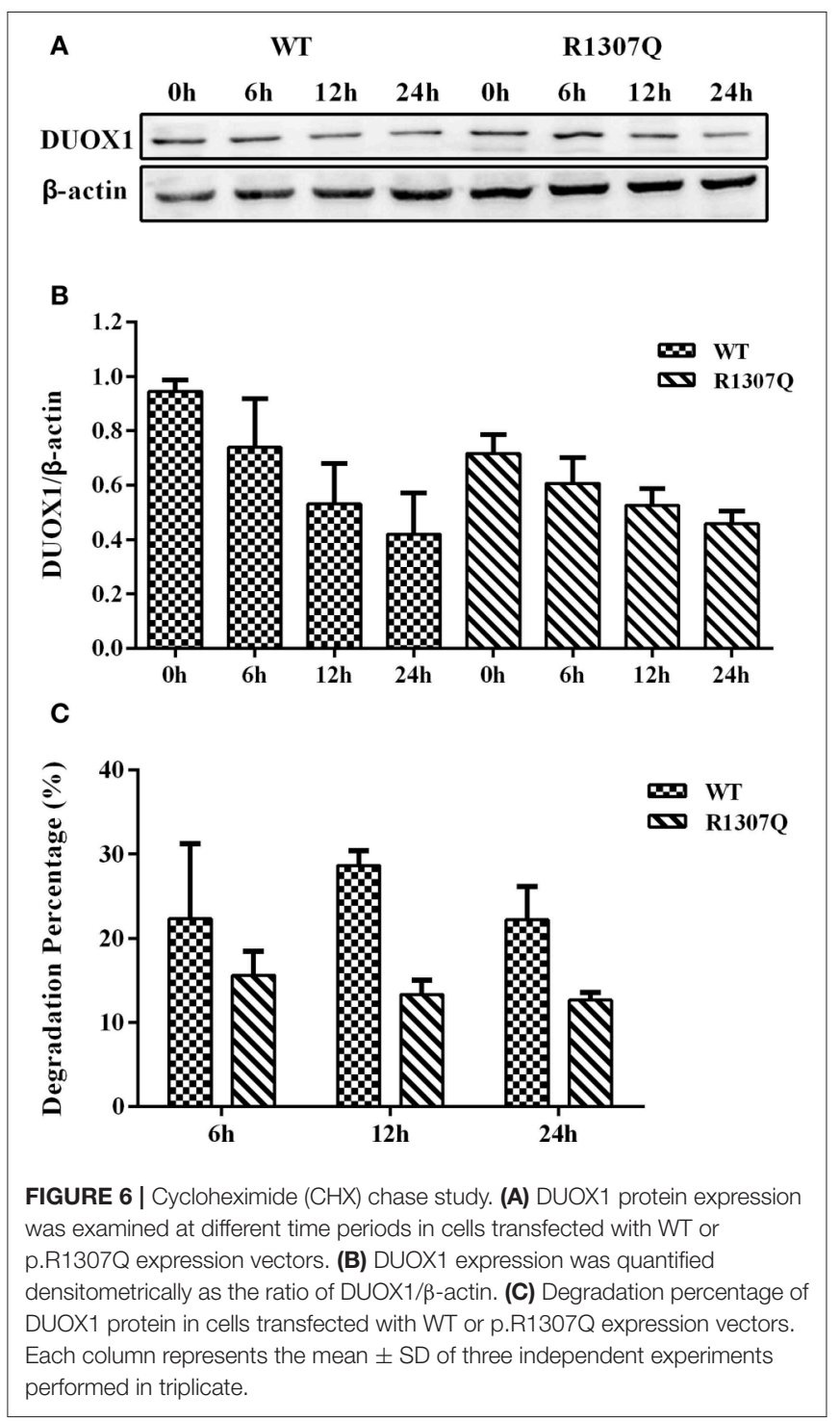

impair the expression of DUOX1 and $\mathrm{H}_{2} \mathrm{O}_{2}$ generation and that intact DUOXA1 is important for the activity of DUOX1. Our study for the first time demonstrates that the DUOX1/DUOXA1 system, when genetically defective, can cause $\mathrm{CH}$.

\section{DATA AVAILABILITY}

The raw data supporting the conclusions of this manuscript will be made available by the authors, without undue reservation, to any qualified researcher.

\section{ETHICS STATEMENT}

Our study was approved by the medical ethics committee of the Affiliated Hospital of Qingdao University and written informed 
consent was signed by each participant or their parents/legal guardians before they participated in the study.

\section{AUTHOR CONTRIBUTIONS}

SL and YG designed the study. WH analyzed the data and drafted the manuscript. $\mathrm{WH}, \mathrm{YZ}$, and $\mathrm{HZ}$ conducted the experiments. FW, HW, and XL collected the sample and analyzed the basic clinical data. SL, PJ, HW, YW, and $\mathrm{XM}$ interpreted the results and provided critical revisions of the manuscript. All authors approved the final version of the manuscript and agreed to be accountable for all aspects of the work.

\section{FUNDING}

This study was supported by the National Natural Science Foundation of China $(81741073,81500689,81470044$, and 81170812) and the National Key Research and Development Program of China under Grant No. 2016YFC1000300 and the

\section{REFERENCES}

1. Rose SR, Brown RS, Foley T, Kaplowitz PB, Kaye CI, Sundararajan S, et al. Update of newborn screening and therapy for congenital hypothyroidism. Pediatrics. (2006) 117:2290-303. doi: 10.1542/peds.2006-0915

2. Park SM, Chatterjee VK. Genetics of congenital hypothyroidism. J Med Genet. (2005) 42:379-89. doi: 10.1136/jmg.2004.024158

3. Afink G, Kulik W, Overmars H, de Randamie J, Veenboer T, van Cruchten A, et al. Molecular characterization of iodotyrosine dehalogenase deficiency in patients with hypothyroidism. J Clin Endocrinol Metab. (2008) 93:4894-901. doi: 10.1210/jc.2008-0865

4. Pohlenz J, Medeiros-Neto G, Gross JL, Silveiro SP, Knobel M, Refetoff S. Hypothyroidism in a Brazilian kindred due to iodide trapping defect caused by a homozygous mutation in the sodium/iodide symporter gene. Biochem Biophys Res Commun. (1997) 240:488-91. doi: 10.1006/bbrc.1997.7594

5. Pfarr N, Borck G, Turk A, Napiontek U, Keilmann A, Muller-Forell W, et al. Goitrous congenital hypothyroidism and hearing impairment associated with mutations in the TPO and SLC26A4/PDS genes. J Clin Endocrinol Metab. (2006) 91:2678-81. doi: 10.1210/jc.2006-0142

6. Abramowicz MJ, Targovnik HM, Varela V, Cochaux P, Krawiec L, Pisarev MA, et al. Identification of a mutation in the coding sequence of the human thyroid peroxidase gene causing congenital goiter. J Clin Invest. (1992) 90:1200-4. doi: $10.1172 /$ JCI115981

7. Medeiros-Neto G, Targovnik H, Knobel M, Propato F, Varela V, Alkmin $\mathrm{M}$, et al. Qualitative and quantitative defects of thyroglobulin resulting in congenital goiter. Absence of gross gene deletion of coding sequences in the TG gene structure. J Endocrinol Invest. (1989) 12:805-13. doi: 10.1007/BF03350067

8. Moreno JC, Bikker H, Kempers MJ, van Trotsenburg AS, Baas F, de Vijlder JJ, et al. Inactivating mutations in the gene for thyroid oxidase 2 (THOX2) and congenital hypothyroidism. N Engl J Med. (2002) 347:95-102. doi: 10.1056/NEJMoa012752

9. Zamproni I, Grasberger H, Cortinovis F, Vigone MC, Chiumello G, Mora S, et al. Biallelic inactivation of the dual oxidase maturation factor 2 (DUOXA2) gene as a novel cause of congenital hypothyroidism. J Clin Endocrinol Metab. (2008) 93:605-10. doi: 10.1210/jc.2007-2020

10. Liu S, Zhang W, Zhang L, Zou H, Lu K, Li Q, et al. Genetic and functional analysis of two missense DUOX2 mutations in congenital hypothyroidism and goiter. Oncotarget. (2016) 9:4366-74. doi: 10.18632/oncotarget.10525

11. Donko A, Peterfi Z, Sum A, Leto T, Geiszt M. Dual oxidases. Philos Trans R Soc Lond B Biol Sci. (2005) 360:2301-8. doi: 10.1098/rstb.2005.1767
Key Research and Development Program of Shandong under Grant No.2016GSF201228.

\section{ACKNOWLEDGMENTS}

We thank all the research subjects and their families for their generous participation in this study. We thank all those colleagues who provided support and help in various forms to this research.

\section{SUPPLEMENTARY MATERIAL}

The Supplementary Material for this article can be found online at: https://www.frontiersin.org/articles/10.3389/fendo. 2019.00526/full\#supplementary-material

Supplementary Figure 1 | The standard calibration curve of $\mathrm{H}_{2} \mathrm{O}_{2}$ release and fluorescence intensity.

Supplementary Figure 2 | The size of the bands of DUOX1 protein in western blot.

12. Dupuy C, Ohayon R, Valent A, Noel-Hudson MS, Deme D, Virion A. Purification of a novel flavoprotein involved in the thyroid NADPH oxidase. Cloning of the porcine and human cdnas. J Biol Chem. (1999) 274:37265-9. doi: $10.1074 /$ jbc.274.52.37265

13. De Deken X, Wang D, Many MC, Costagliola S, Libert F, Vassart G, et al. Cloning of two human thyroid cDNAs encoding new members of the NADPH oxidase family. J Biol Chem. (2000) 275:23227-33. doi: 10.1074/jbc.M000916200

14. Grasberger, H. Defects of thyroidal hydrogen peroxide generation in congenital hypothyroidism. Mol Cell Endocrinol. (2010) 322:99-106. doi: 10.1016/j.mce.2010.01.029

15. Morand S, Ueyama T, Tsujibe S, Saito N, Korzeniowska A, Leto TL. Duox maturation factors form cell surface complexes with Duox affecting the specificity of reactive oxygen species generation. FASEB J. (2009) 23:1205-18. doi: 10.1096/fj.08-120006

16. Hoste C, Rigutto S, Van Vliet G, Miot F, De Deken X. Compound heterozygosity for a novel hemizygous missense mutation and a partial deletion affecting the catalytic core of the $\mathrm{H}_{2} \mathrm{O}_{2}$-generating enzyme DUOX2 associated with transient congenital hypothyroidism. Hum Mutat. (2010) 31:E1304-19. doi: 10.1002/humu.21227

17. Hulur I, Hermanns P, Nestoris C, Heger S, Refetoff S, Pohlenz J, et al. A single copy of the recently identified dual oxidase maturation factor (DUOXA) 1 gene produces only mild transient hypothyroidism in a patient with a novel biallelic DUOXA2 mutation and monoallelic DUOXA1 deletion. J Clin Endocrinol Metab. (2011) 96:E841-5. doi: 10.1210/jc.2010-2321

18. Liu C, Zhang GQ, Zang YC, Wang F, Han MM, Han WX, et al. Genetic screening of solute carrier family 5 member 5 (SLC5A5) gene in Chinese Han congenital hypothyroidism patients with goiter. Int J Clin Exp Pathol. (2016) 9:9461-6. Available online at: http://www.ijcep.com/files/ijcep0028085. pdf

19. De Deken X, Wang D, Dumont JE, Miot F. Characterization of ThOX proteins as components of the thyroid $\mathrm{H}(2) \mathrm{O}(2)$-generating system. Exp Cell Res. (2002) 273:187-96. doi: 10.1006/excr.2001.5444

20. Lambeth JD, Cheng G, Arnold RS, Edens WA. Novel homologs of gp91phox. Trends Biochem Sci. (2000) 25:459-61. doi: 10.1016/S0968-0004(00)01658-3

21. Krause KH. Tissue distribution and putative physiological function of NOX family NADPH oxidases. Jpn J Infect Dis. (2004) 57:S28-9. Available online at: http://www0.nih.go.jp/JJID/57/S28.html

22. Corvilain B, Collyn L, Van Sande J, Dumont JE. Stimulation by iodide of $\mathrm{H}(2) \mathrm{O}(2)$ generation in thyroid slices from several species. Am J Physiol Endocrinol Metab. (2000) 278:E692-9. doi: 10.1152/ajpendo.2000.278.4.E692 
23. Cardoso LC, Martins DC, Figueiredo MD, Rosenthal D, Vaisman M, Violante $\mathrm{AH}$, et al. $\mathrm{Ca}(2+) /$ nicotinamide adenine dinucleotide phosphatedependent $\mathrm{H}(2) \mathrm{O}(2)$ generation is inhibited by iodide in human thyroids. J Clin Endocrinol Metab. (2001) 86:4339-43. doi: 10.1210/jcem.8 6.9.7823

24. O'neill S, Brault J, Stasia MJ, Knaus UG. Genetic disorders coupled to ROS deficiency. Redox Biol. (2015) 6:135-56. doi: 10.1016/j.redox.201 5.07.009

25. Grasberger H, Refetoff S. Identification of the maturation factor for dual oxidase. Evolution of an eukaryotic operon equivalent. J Biol Chem. (2006) 281:18269-72. doi: 10.1074/jbc.C600095200

26. Yi RH, Zhu WB, Yang LY, Lan L, Chen Y, Zhou JF, et al. A novel dual oxidase maturation factor 2 gene mutation for congenital hypothyroidism. Int J Mol Med. (2013) 31:467-70. doi: 10.3892/ijmm.2012.1223

27. Donko A, Ruisanchez E, Orient A, Enyedi B, Kapui R, Peterfi $\mathrm{Z}$, et al. Urothelial cells produce hydrogen peroxide through the activation of Duoxl. Free Radic Biol Med. (2010) 49:2040-8. doi: 10.1016/j.freeradbiomed.2010.09.027

28. Grasberger H, Refetoff S. Genetic causes of congenital hypothyroidism due to dyshormonogenesis. Curr Opin Pediatr. (2011) 23:421-8. doi: 10.1097/MOP.0b013e32834726a4

29. Grasberger H, De Deken X, Miot F, Pohlenz J, Refetoff S. Missense mutations of dual oxidase 2 (DUOX2) implicated in congenital hypothyroidism have impaired trafficking in cells reconstituted with DUOX2 maturation factor. Mol Endocrinol. (2007) 21:1408-21. doi: 10.1210/me.2007-0018

30. Tan MY, Huang YL, Li B, Jiang X, Chen QY, Jia XF, et al. Characteristics of DUOXA2 gene mutation in children with congenital hypothyroidism. Zhongguo Dang Dai Er Ke Za Zhi. (2017) 19:59-63. Available online at: http://www.zgddek.com/EN/article/ downloadArticleFile.do?attachType=PDF\&id $=14148$

31. Wang F, Lu K, Yang Z, Zhang S, Lu W, Zhang L, et al. Genotypes and phenotypes of congenital goitre and hypothyroidism caused by mutations in dual oxidase 2 genes. Clin Endocrinol (Oxf). (2014) 81:452-7. doi: $10.1111 /$ cen.12469

Conflict of Interest Statement: The authors declare that the research was conducted in the absence of any commercial or financial relationships that could be construed as a potential conflict of interest.

Copyright (c) 2019 Liu, Han, Zang, Zang, Wang, Jiang, Wei, Liu, Wang, Ma and Ge. This is an open-access article distributed under the terms of the Creative Commons Attribution License (CC BY). The use, distribution or reproduction in other forums is permitted, provided the original author(s) and the copyright owner(s) are credited and that the original publication in this journal is cited, in accordance with accepted academic practice. No use, distribution or reproduction is permitted which does not comply with these terms. 